A N N A L E S Annales de Bretagne et des Pays de l'Ouest

\title{
Genre et pouvoir à la lumière de l'histoire du féminisme
}

\section{Christine Bard}

\section{(2) OpenEdition}

\section{Journals}

Édition électronique

URL : http://journals.openedition.org/abpo/1743

DOI : $10.4000 / a b p o .1743$

ISBN : 978-2-7535-1482-9

ISSN : 2108-6443

Éditeur

Presses universitaires de Rennes

Édition imprimée

Date de publication : 20 juin 2001

Pagination : $71-84$

ISBN : 978-2-86847-635-7

ISSN : 0399-0826

\section{Référence électronique}

Christine Bard, «Genre et pouvoir à la lumière de l'histoire du féminisme », Annales de Bretagne et des

Pays de l'Ouest [En ligne], 108-2 | 2001, mis en ligne le 20 juin 2003, consulté le 21 avril 2019. URL

http://journals.openedition.org/abpo/1743 ; DOI : 10.4000/abpo.1743 


\title{
AN WA BRETAGNE PAYS DE L'OUEST \\ Annales de Bretagne et des Pays de l'Ouest
}

Numéro 108-2 (2001)

108-2

Christine BARD

\section{Genre et pouvoir à la lumière de l'histoire du féminisme}

\begin{abstract}
Avertissement
Le contenu de ce site relève de la législation française sur la propriété intellectuelle et est la propriété exclusive de l'éditeur.

Les œuvres figurant sur ce site peuvent être consultées et reproduites sur un support papier ou numérique sous réserve qu'elles soient strictement réservées à un usage soit personnel, soit scientifique ou pédagogique excluant toute exploitation commerciale. La reproduction devra obligatoirement mentionner l'éditeur, le nom de la revue, l'auteur et la référence du document.

Toute autre reproduction est interdite sauf accord préalable de l'éditeur, en dehors des cas prévus par la législation en vigueur en France.
\end{abstract}

\section{revues.org}

Revues.org est un portail de revues en sciences humaines et sociales développé par le Cléo, Centre pour l'édition électronique ouverte (CNRS, EHESS, UP, UAPV).

Référence électronique

Christine BARD, « Genre et pouvoir à la lumière de I'histoire du féminisme », Annales de Bretagne et des Pays de l'Ouest [En ligne], 108-2 | 2001, mis en ligne le 20 juin 2003. URL : http://abpo.revues.org/1743

$\mathrm{DOI}$ : en cours d'attribution

Éditeur : Presses universitaires de Rennes

http://abpo.revues.org

http://www.revues.org

Document accessible en ligne sur : http://abpo.revues.org/1743

Ce document est le fac-similé de l'édition papier.

(c) Presses universitaires de Rennes 


\title{
Genre et pouvoir à la lumière de l'histoire du féminisme
}

\author{
Christine BARD \\ Maître de conférences en histoire contemporaine \\ Université d'Angers et Institut Universitaire de France
}

\begin{abstract}
À l'invitation du groupe d'histoire des femmes de l'Université de Rennes 2, une réflexion sur "genre et pouvoir» m'a été proposée. Sur un thème aussi vaste et aussi complexe, je ne peux apporter que quelques idées et interrogations issues de mes recherches sur l'histoire des féminismes, des antiféminismes, des transgressions des codes vestimentaires (à travers les garçonnes et les femmes travesties). L'exercice tendu vers la synthèse suppose que l'on se déleste des matériaux empiriques qui font l'essentiel du travail (et du bonheur) de l'historien-ne. Cette contribution n'est pas académique : j'ai voulu réfléchir sans rouvrir livres et dossiers aux questions posées par les organisatric es et organisateurs de cette journée d'études rennaise. Les réflexions qui suivent n'ont pas non plus d'ambition théorique; d'autres historiennes ont, à partir de leurs connaissances sur l'histoire du féminisme, évolué vers une approche plus philoso phique des rapports genre-pouvoir ${ }^{1}$. Parmi les historiennes $d u$ féminisme, je me sens de celles qui restent attachées à l'histoire des femmes ${ }^{2}$. D'autres, se situant plutôt dans une histoire du genre, tendent vers une histoire politique (plus exactement de la pensée politique) où la critique féministe devient centrale (Michèle Riot-Sarcey sur la démocratie, ou celui de Joan Scott sur le républicanisme et sa théorie universelle des droits de l'homme ${ }^{3}$ ) ou vers la sociologie politique ${ }^{4}$. Mon propos est
\end{abstract}

1. Voir entre autres, BUTLER, Judith et SCOTT, Jo an W., Fe minists theorize the political, London, New York, Routledge, 1992 ou l'introduction de Michèle RIOT-SARCEY à Femmes pouvoirs, Paris, Kimé, 1993.

2. Sans doute (en partie) par attachement aux connotations militantes de cette expression, explicitées dans l'ouvrage dédié à la mémoire de Marie-France BRIVE, Les Femmes sujets d'histoire, sous la direction de CORRADIN, Irène et MARTIN, Jacqueline, Toulo use, Presses universitaires de Mirail, 1999.

3. RIOT-SARCEY, Michèle, La Démocratie à l'épreuve des femmes. Trois figures critiques du pouvoir 1830-1848, Albin Michel, 1994 et ScOTT, Jo an W., La Citoyenne paradoxale. Les féministes françaises et les droits de l'homme, Albin Michel, 1998. 
simplement d'examiner les notions (et non les concepts) qui ont balisé mes recherches en donnant quelques exemples de leur application.

\section{Une première difficulté: la polysémie du «genre»}

"Genre». Cette notion est désormais présente dans une littérature assez fournie. Son emploi est de plus en plus fréquent, mais sa définition n'est pas univoque. Nul n'a le privilège - et l'on peut s'en réjo uir - d'en fixer le sens ${ }^{5}$. Beaucoup d'entre nous, dans la communauté historienne, «bricolent» avec le genre. Bien que l'on puisse considérer le bricolage comme une discipline noble, le flou - que bien des sociologues nous reprochent - représente une difficulté que nous avons à affronter.

Le genre est souvent utilisé comme synonyme d'identité sexuelle. Il est parfo is employé à la place de "femmes " ou "hommes", ce qui a pour effet de masquer les acteurs/actrices par une notion abstraite. Est-ce parce qu'elle est politiquement moins gênante que sexe ${ }^{6}$ ? Serait-elle alors une sorte d'euphémisme? L'expression "rapports sociaux de sexe " (et non de genre), fréquemment utilisée en sociologie, a le mérite de la clarté. En revanche, pour décrire la conscience identitaire, il me semble que l'emploi du mot genre convient bien (j'y reviendrai plus tard).

Le genre est appréhendé, par l'histoire des femmes faisant sienne les analyses beauvoiriennes, comme une construction sociale qui est surtout vue comme une contrainte. L'histoire du travail, de l'éducation ou du droit apporte beaucoup dans ce domaine. Plus généralement, la normativité de genre est une structure fondamentale des sociétés occidentales o ù règne la bipolarité des sexes et bipolarité des genres et où le genre doit coüncider exactement avec le sexe ${ }^{7}$. On pourrait ajouter avec ce que l'on croit savoir du sexe, avec nos représentations mouvantes du sexe biologique tel que les scientifiques le définissent ${ }^{8}$. L'analyse (d'origine féministe), selon laquelle cette bipolarité des genres est l'habillage de la hiérarchie des sexes semble aujourd'hui largement admise.

4. L'o uvrage de MUEL-DREYFus, Francine, Vichy et l'éternel féminin, Seuil, 1986, co mme l'indique son sous-titre, "contribution à une sociologie politique de l'ordre des corps", est un des rares exemples pour la France d'approche sociologique (inspirée des travaux de Pierre Bourdieu) de l'histoire du genre.

5. Sur l'émergence de l'histoire du genre, fille - parfois ingrate - de l'histoire des femmes, voir THÉBAUD, Françoise, Écrire l'histoire des femmes, Fontenay-aux-Roses, ENS Saint-Cloud, 1998.

6. C'est ce que suggère la sociologue féministe Christine DELPHY dans "Penser le genre : quels problèmes? ", HuRTiG, Marie-Claude, KAI, Michèle, RoucH, Hélène, (dir.), Sexe et genre. De la hiérarchie entre les sexes, éd. du CNRS, 1991, p. 89-102.

7. MATHEU, Nicole-Mathieu, L'anatomie politique. Catégorisations et idéologies du sexe, Côté-femmes, 1991.

8. LAQUEUR, Thomas, La Fabrique du sexe. Essai sur le corps et le genre en Occident, Gallimard, 1990. 
Jusqu'à présent, le mot genre a un pouvoir plutôt descriptif. En histoire, nous observons comment se construit, se reconstruit le genre, sans nécessairement nous engager dans les débats théoriques. Lorsque nous utilisons le genre comme outil d'analyse, c'est souvent pour rechercher l'effet de genre : pour dévoiler un déterminisme social.

Mais le genre ne se réduit pas à une contrainte. On peut le voir, d'une manière plus dynamique, comme un enjeu de pouvoir (nommer, classer, hiérarchiser), qui suscite des controverses, des résistances. Il est aussi parfois considéré comme une mascarade, ludique et érotique. Encouragés par la théorie de Freud sur la bisexualité psychique des individus, les psychanalystes s'interrogent sur la fluidité du genre, s'intéressent, par exemple, à la présence du "féminin " chez des individus de sexe masculin, ou du "masculin " chez des individus de sexe féminin ${ }^{9}$. La particularité $\mathrm{du} \mathrm{XX}^{\mathrm{e}}$ siècle est d'être très bavard sur ces circulations, d'abord en termes rudes, médico-sexologiques (inversions), politiques (par exemple, insistance des antiféministes et/ou de l'extrême droite sur la masculinisation des femmes et l'effémination des hommes) et, de plus en plus, en termes positifs et libératoires, ce dernier discours, issu des mouvements des années 1970, notamment féministes et homosexuels, trouvant aujourd'hui des développements très intéressants dans la pensée queer et l'ouvrage marquant de la philo so phe Judith Butler, Gender Trouble ${ }^{10}$.

\section{En quoi la prise en compte du genre peutelle soulever de nouveaux questionnements dans l'analyse du pouvoir?}

Examinons d'abord la genèse de cette question : le pouvoir masculin ne s'est généralement pas donné la peine de justifier sa nature unisexuée (il n'est question ici que de la sphère publique, dans le monde occidental, et à l'épo que contemporaine). Son intérêt était au contraire de naturaliser l'exclusion des femmes, de faire comme si elle allait de soi ${ }^{11}$. Ce sont les féministes qui ont posé la question et forgé cette notion de "genre".

9. Sur les ambiguités de ces théories, voir Kofman, Sarah, L'Énigme de la femme. La femme dans les textes de Freud, Galilée/ Le Livre de Poche, 1994.

10. ButLER, Judith, Gender Trouble. Fe minism and the subversion of identity, New York, Routledge, 1990 (un "classique " qui peut rivaliser avec Le Deuxième sexe de Simone de Beauvoir, toujours pas traduit en français). De même, la queer theory qui se développe depuis plus de dix ans aux États-Unis et dans le monde anglophone plus généralement n'a qu'un très faible écho en France. Le cloisonnement des disciplines dans le monde ac adémique hexago nal suffit-il à expliquer la méconnaissance de ces travaux?

11. Exemple qui pourrait être célèbre entre to us : la loi sur le «suffrage universel»en 1848 mentionne les droits des Français majeurs sans préciser qu'ils sont de sexe masculin et sans ajouter que les Françaises ne disposent pas de ce droit. Hubertine Auclert et d'autres suffragistes tenteront d'ailleurs, quelques dizaines d'années plus tard, de s'inscrire sur les listes électorales en jouant de l'imprécision linguistique (un masculin pluriel peut en effet être mixte, «le masculin l'emportant sur le féminin ", pour nos grammairiens). 
Utiliser le mot genre en histoire politique permet de nommer le genre qui ne l'était pas, de désuniversaliser le genre masculin, par exemple en parlant de mono pole masculin du pouvoir. Ce qui ouvre to ut un champ de recherche sur le rôle du politique dans la construction du masculin (socialisation et sociabilité). Ce qui nous fait découvrir aussi du genre féminin en politique. Par exemple, jusqu'en 1940, le féminisme est fréquemment présenté comme un "parti » (d'ailleurs présent aux élections, avec un programme généraliste qui va au-delà de la défense des droits des femmes). Ce sont ces observateurs contemporains commentant l'existence de ce "parti féministe " qui sont dans le juste, et non les historiens $d u$ politique travaillant avec une définition étroitement institutionnelle. Plus généralement, on ne peut négliger, surto ut à partir de la loi de 1901, l'associationnisme féminin que l'on pourrait qualifier de parapolitique.

Notre vision du pouvoir doit s'élargir aux formes de résistances. Pour agir en contre-pouvoir, il faut d'abord disposer d'un certain pouvoir. II faut aussi que les circonstances historiques permettent l'émergence de ce pouvoir. S'agissant du féminisme - défini comme mouvement collectif aspirant à transformer les relations entre les sexes, avec, à l'horizon leur égalité dans le droit et dans les faits - ces conditions d'émergence et de développement sont variées : elles sont intellectuelles et politiques mais aussi sociales (éducation, professionnalisation, légitimation - même partielle - de modes de vie alternatifs à la famille traditionnelle, etc.). Étant entendu que ces conditions favorables, les pionnières de l'émancipation des femmes ont contribué à les créer (dans quelle mesure? le débat n'est pas clos).

Que ses campagnes soient offensives ou défensives, le féminisme est avant tout une forme de résistance. Cette résistance se décline pour beaucoup sur un mode optimiste : le partage équitable du pouvoir (pour certaines aujourd'hui la parité) devrait rendre cette résistance inutile. Pour d'autres, sur un mode pessimiste : les risques constants de régressions, les capacités du patriarcat à concéder des droits pour se perpétuer, à inventer des modes de coercition des femmes toujours plus subtils, intériorisés, presque invisibles, condamneraient les féministes à une résistance perpétuelle.

Mais le féminisme n'est pas seulement résistance, il est aussi projet(s). Et donc réflexion sur le pouvoir, sur le politique, ce qui va bien au-delà d'une exigence de répartition équitable du pouvoir entre les sexes. Ainsi, on peut résumer le projet des mouvements féministes en France de 1914 à 1940 à une volonté d'intégration des femmes dans la Cité ${ }^{12}$. Mais dans quelle Cité ? Sur cette question fondamentale, les féministes sont divisées.

12. BARD, Christine, Les Filles de Marianne. Histoire des féminismes 1914-1940, Fayard, 1995. Cet ouvrage est issu de ma thèse dont le sous-titre était "Vers l'intégration des femmes dans la Cité ». 
Ces divergences prennent un tour assez dramatique dans les années 1930: toutes n'ont pas le réflexe antifasciste et/ou républicain et/ou patriotique. Si l'horizon du féminisme se borne au droit de vote, alors, prenant acte de la trahison républicaine et du piège de l'adhésion à l'Union sacrée, l'ultrapacifisme se trouve légitimé, pour ne pas se laisser piéger une seconde fois ${ }^{13}$. La question du projet féministe est posée encore en bien d'autres occasions. La nomination de conseillères municipales, décidée par Pétain, pose le problème : pour les unes - les plus modérées - c'est une victoire que les femmes doivent fêter; pour les autres, ce qui l'emporte est un jugement plus global sur le nouveau régime qui bafoue les libertés individuelles et collectives et supprime les élections. Pour les résistantes ayant une sensibilité féministe, le caractère masculin du pouvoir de l'occupant et de l'État français est-il décisif? Évidemment non. Dans les grandes tourmentes du premier $x^{e}$ siècle, la conscience de genre s'estompe, atténuée par des appartenances plus décisives: être française, étrangère, juive, communiste, ouvrière, etc. L'histoire même du féminisme révèle une conscience à éclipses et d'autant plus éclipsée que le féminisme perd de sa force ${ }^{14}$.

\section{La notion de conscience de genre/de sexe}

Ne faudrait-il pas d'ailleurs parler de conscience de sexe ? Par exemple Madeleine Pelletier (1874-1939), grande figure du féminisme radical et partisane de la virilisation des femmes, a une conscience de sexe tout en refusant - et en méprisant - le genre féminin ${ }^{15}$. Par conscience de genre, j'entends donc conscience d'appartenir à un groupe humain discriminé. C'est-à-dire que je procède comme les marxistes avec la conscience de classe : un "jaune", même lucide sur son statut d'ouvrier, n'a pas de conscience de classe car il admet et légitime la hiérarchie sociale. Autant dire qu'avec cette définition, la conscience de genre est rare et qu'elle se confond le plus souvent avec une posture féministe de dénonciation du sort fait aux femmes. Michelle Perrot remarque qu'aujourd'hui, «il existe un féminisme latent. Il y a de plus en plus chez les femmes "une conscience de genre", liée au constat qu'être femme n'est pas neutre et implique des contraintes et une inégalité particulière ${ }^{16}$ ». Cette impression est sans doute juste (elle n'est pas encore vérifiée par des enquêtes approfondies). Prudente, l'historienne ento ure de guillemets l'expression «conscience de genre » et suggère une définition féministe.

13. Telle est l'attitude de la Ligue française du droit des femmes, la plus radicale des associations réformistes.

14. Jévoque ici la période de "creux de la vague", de la fin des années 1930 aux années 1950 (Cf. CHAPERON, Sylvie, Les Années Be auvoir, Fayard, 2000).

15. Voir mon article sur "La virilisation des femmes et l'égalité des sexes" dans BARD, Christine, (dir.), Madeleine Pelletier (1874-1939). Logique et infortunes d'un combat pour l'égalité, Côté-femmes, 1992, p. 91-108.

16. "Le $\mathrm{XX}^{\mathrm{e}}$ siècle a-t-il libéré les femmes? ", La revue de la CFDT, $\mathrm{n}^{\circ} 28$, février 2000, p. 6. 
La notion de "conscience" pose des problèmes de méthode et de sources. En travaillant sur un gros corpus de textes féministes, j'ai souvent rencontré des proclamations identitaires particulièrement élaborées. L’enjeu est énorme pour les féministes : la nature féminine ayant été définie par d'autres (des hommes), il faut riposter, contester la définition patriarcale du genre féminin, en proposer d'autres. Il s'agit avant to ut de prendre un pouvoir essentiel, celui de l'autodéfinition. Il faut ensuite analyser ces autodéfinitions identitaires à visée militante : leur philogynie, leur degré de rupture avec la définition dominante, leur insistance sur (ou leur relativisation de) la composante maternelle de l'identité féminine, leurs mythes (la non-violence féminine, par exemple). Elles rompent rarement avec le naturalisme ambiant, même s'il existe des tentatives de déconstruction à la Beauvoir bien avant le Deuxième Sexe.

Aussi séduisantes que certaines de ces définitions puissent paraître, elles méritent un examen critique : que penser de Virginia Woolf définissant dans Trois Guinées les femmes comme des "parias» à qui elle prête une lucidité particulière sur le monde? Ce serait alors un rapport d'exclusion au pouvoir qui constituerait le genre des femmes. Vision trop schématique : certaines femmes ont eu ou ont du pouvoir. Par ailleurs, celles qui en sont radicalement privées n'ado ptent pas nécessairement une posture contestataire.

Nous n'en sommes, je crois, qu'au tout début de la réflexion sur la conscience (ou l'inconscience) identitaire des femmes. Et cela pose des défis méthodologiques passionnants. Leurs prolongements politiques sont aussi importants : la parité pose aujourd'hui de manière assez brutale cette question ${ }^{17}$. Sa popularité indique-t-elle un réveil de la conscience de genre? Ses adversaires parmi les féministes ne la ressentent-elles pas comme une assignation identitaire assez violente? Les antiféministes ne trouvent-ils pas là l'occasion de déno ncer une vision " politiquement correcte " des femmes en politique supposées plus pures, moins violentes et donc moins attaquables que leurs collègues masculins? La plupart des "paritaristes" ont voulu la réparation d'une injustice sans présupposer une quelconque essence du féminin en politique. Ces critiques, elles (et ils) s'y attendaient. On peut supposer que ces craintes n'auront guère plus de conséquences que les supputations sur le futur comportement des électrices, avant 1945. Pourquoi une autre forme d'assignation identitaire, cette fois-ci par défaut, l'exclusion des femmes du service militaire, choquait-elle moins?

Comment mesurer la conscience de genre? Quelles sources utiliser? Comment distinguer effet de genre et conscience de genre? Prenons l'exemple du vote conservateur des électrices de 1945 à 1981. Il est consi-

17. Voir les deux numéros spéciaux de Nouvelles questions féministes: La parité "pour», novembre 1994 et La parité «contre», mai 1995, ainsi que l'utile synthèse de MosSUZ-LAVAU, Janine, Fe mmes / hommes pour la parité, Presses de sciences po, 1998. 
déré par les politologues comme un effet de genre (découlant d'une pratique religieuse plus forte chez les femmes et du poids des femmes au foyer), mais les tendances progressistes de l'électorat féminin depuis vingt ans sont au contraire analysées comme l'expression d'une conscience de genre : les femmes voteraient désormais en tenant compte de leurs intérêts propres en tant que femmes ${ }^{18}$. C'est sans doute vrai : la conscience identitaire est probablement corrélée à la conscience féministe. Plus le féminisme est influent, plus cette conscience de genre est forte. Mais pourquoi ne pas explorer la «conscience de genre" des électrices d'avant 1981 ?

\section{Comment expliquer la fragilité de la conscience de genre?}

Le déni ou l'absence de conscience de genre peut correspondre à un réflexe inconscient. $J$ allais dire un réflexe de survie, ce qui est excessif, sauf si l'on admet les ravages psychologiques provoqués par les violences masculines, si longtemps sous-estimées dans le monde universitaire. Il s'agirait donc d'une résistance paradoxale à la domination. Une stratégie d'échappement: je ne suis pas là, je prétends ne pas être là où vous voulez m'atteindre. Il en est un exemple (terrible) très courant : il est souvent donné d'entendre des femmes dire "je ne suis pas une «femme", je suis un être humain".

Le terme de conscience travestie a été proposé par une spécialiste de la réception des films qui cherchait à expliquer pourquoi la majorité des spectatrices accepte, s'habitue au spectacle des violences contre les femmes sur grand écran ${ }^{19}$. Dans la salle obscure, mieux vaut s'identifier aux hommes qu'aux femmes. L'expression me semble mieux adaptée que la «fausse conscience» employée par la sociologue féministe Colette Guillaumin pour décrire l'aliénation. Ce terme est aussi utilisé par la sociologue féministe Christine Delphy, pour qualifier les femmes antiféministes :

«L'antiféminisme des femmes diffère radicalement de l'antiféminisme des hommes; il lui est même diamétralement opposé. Ce qui est racisme chez l'oppresseur est haine de soi chez l'opprimée. Il est normal que les femmes soient antiféministes; c'est le contraire qui serait étonnant. Et la prise de conscience, le «devenir-féministe " n'est pas une Pentecôte soudaine et brutale; la conscience n'est pas acquise une fois et une fois pour toutes; c'est un processus long et jamais terminé, douloureux de surcroît, car c'est une lutte de tous les instants contre les «évidences » : la vision idéolo gique du monde, et contre soi. La lutte contre la haine de soi n'est jamais terminée. Il n'y a donc pas de rupture abrupte entre les femmes féministes et les femmes "antifé-

18. Mossuz-Lavau, Janine, "Le vote des femmes en France (1945-1993) ", Revue française de science politique, ao ût 1993.

19. DoAnE, Mary Ann, "Film and the Masquerade: Theorising female Spectator", Sereen, n³-4, septembre-oc to bre 1982, p. 74-88. 
ministes", mais un continuum de points de vue sur une même situation. Car, quelles que soient leurs "opinions", les femmes sont opprimées. Leur antiféminisme étant a) un obstacle à la prise de conscience de leurs intérêts objectifs et b) plus directement le reflet de leur oppression dans leur subjectivité est donc l'un des moyens du maintien de cette oppression.

Aussi, tandis que l'antiféminisme des hommes fait partie de l'oppression exercée, l'antiféminisme des femmes fait-il partie de l'oppression subie. Les féministes ne peuvent en aucun cas considérer sur le même pied les hommes antiféministes et les femmes antiféministes, ni appeler ces dernières des ennemies. Elles ne sont pas séparées de nous par des intérêts objectifs mais par une fausse conscience, et encore celleci ne nous sépare-t-elle pas vraiment: car nous l'avons eue, l'avons encore en partie; c'est notre ennemie commune. Quand nous luttons contre leurs «opinions", nous ne luttons pas contre elles, mais contre cette ennemie commune, donc pour elles et pour nous ${ }^{20}$ ».

La plupart des femmes à la recherche d'une intégration dans un milieu masculin (cadres, vie politique, syndicalisme) adoptent une stratégie de neutralisation de leur genre : jusqu'à une date récente, leur opposition à la féminisation des noms de métiers, des titres et des fonctions le prouve, tout comme leurs choix vestimentaires, sans doute aussi leur manière de parler et leur gestuelle. En d'autres termes, l'évitement d'une conscience de genre correspondrait à une adaptation au monde patriarcal. Sans doute aussi à un déni de la domination masculine que l'on détecte dans le syndrome de la self made woman: "j'y suis bien arrivée, moi!». Il est difficile dans ce cas de faire la part du conscient et de l'inconscient.

Affirmer une conscience de genre, surtout en France, c'est s'exclure de l'universel et s'abîmer dans le spécifique (ou le "communautarisme »). Question brûlante dans la vie politique : ne pas tomber dans le piège des commissions féminines dépourvues de pouvoir ou dans la répartition sexuée des postes (aux femmes, les affaires de femmes, la santé, le social, l'enfance, etc.). Question cruciale aussi pour les créatrices et les intellectuelles, si elles veulent participer au banquet. Le danger est si grand que même les cinéastes les plus féministes - Coline Serreau, par exemple refusent que leurs films soient qualifiés de «films de femme(s)» ${ }^{21}$. Est-ce l'amalgame possible entre conscience de genre et stéréotype féminin dévalué qui est à craindre? Comme il me semble établi que la conscience de genre est douloureuse et socialement couteuse, il faut sans doute essayer de mieux cerner les bénéfices qui s'attachent à une conscience de genre revendiquée.

20. DelPHY, Christine, «Nos amis et nous. Fondements cachés de quelques discours pseudo-féministes", Questions féministes, novembre 1977, rééd. dans L'Ennemi principal, t. 1. Économie politique du patriarcat, Paris, Syllepse, 1998, p. 191.

21. Rollet, Brigitte, Coline Serre au, Manchester University Press, 1998. 


\section{Être féministe et avoir du pouvoir}

Être féministe est une prise de pouvoir. Le terme anglais intraduisible d'empowerment conviendrait mieux. Les militantes conquièrent une liberté personnelle, des savoir-faire (écrire, prendre la parole en public), une manière de penser le collectif qui donne sens à des événements personnels et des sentiments intimes, et se donnent une généalo gie héroïque de femmes rebelles, exceptionnelles. Au niveau collectif aussi, elles gagnent du pouvoir: dans la contestation pure souvent, mais parfois aussi dans la participation au pouvoir (ce que l'on appelle parfois le «féminisme d'État», depuis le précédent créé par la no mination de Françoise Giroud en 1974).

Mais une femme qui accède à ce pouvoir est-elle encore une «femme»? N'est-elle pas d'emblée masculinisée par ses détracteurs? N'est-elle pas elle-même en porte-à-faux par rapport à son genre qui est, par définition, dépourvu de pouvoir? Ne se coupe-telle pas de la masse des femmes (dont elle se prétend le porte-parole) un peu comme le syndicaliste autour de la table de négociation ou dans son bureau à la bourse du travail se coupe de la masse ouvrière? Quelle part de fiction dans le "nous les femmes" des féministes? Dans quelle mesure peut-on aussi parler d'un pouvoir des féministes sur les femmes? L'exemple le plus frappant est sans doute celui des féministes réformistes et modérées de l'entre-deux-guerres qui ont demandé puis soutenu la loi de 1920 durcissant la répression de l'avortement et interdisant les moyens de contraception et leur publicité. Ce faisant, elles sont en contradiction avec les aspirations de ces femmes et de ces hommes assez no mbreux pour que la France soit durement qualifiée de malthusienne et qui, malgré les obstacles, limitent le nombre de leurs enfants.

Et quid du pouvoir entre féministes? Oppositions idéologiques et querelles de personnes ont considérablement affaibli le mouvement en France ${ }^{22}$. "Des généraux sans troupes ", lâchait méchamment Madeleine Pelletier, constatant que chacune voulait créer sa chapelle pour mieux établir son pouvoir. Car il y a effectivement là un pouvoir à prendre. Ces conflits de pouvoir entre féministes sont-ils "genrés »? Prennent-ils une forme spécifique liée à l'homosocialité du mouvement? Je n'en ai pas l'impression pour le féminisme de la première vague, dont l'organisation quand il s'agit de grandes associations réformatrices ressemble au modèle masculin (associatif, partisan). En revanche, le radicalisme de la deuxième vague met en cause ce modèle. En ces années post-68, le centralisme démocratique mais aussi les groupes dits d'avant-garde sont contestés. Les nouvelles féministes déclinent à leur manière l'autoges-

22. Pour une analyse de ces tensions depuis les années 1970, cf. Lous, MarieVic toire, "Éléments pour une critique des rapports des féministes françaises au pouvoir ", dans Viennot Eliane, (dir.), La démocratie «à la française» ou les femmes indésirables, Publications de l'université de Paris 7 - Denis Diderot, 1996, p. 91-107. 
tion, sans revendiquer ce terme. Cela n'aura qu'un temps puisque dès le milieu des années 1970, les associations structurées prennent une importance croissante. Pendant quelques années auront été expérimentés des rapports nouveaux entre les "femmes en mouvement". Ils se veulent strictement égalitaires, "sororels", et bannissent même les notions de représentation ou de délégation. Une seule instance de pouvoir : l'assemblée générale où «la parole est à tout le monde, mais elle doit être prise, d'autorité ${ }^{23}$ ". Comme le reconnaissent volontiers aujourd'hui la plupart des actrices de cette période, l'absence to tale de régulation des rapports de pouvoir n'est pas en mesure de supprimer ces derniers et corse les conflits.

\section{Le féminisme à la lueur du «genre»}

Le concept de genre n'est pas le plus opératoire pour comprendre le féminisme de la première vague. On peut le faire intervenir dans la psychogenèse de l'engagement militant, en voir les traces dans une certaine timidité, un certain sérieux qui est au fond le propre de beaucoup d'outsiders, doutant de leur légitimité. On le retrouve sans doute aussi dans des préoccupations politiques en prise avec des expériences féminines (la maternité en tout premier lieu). Mais cela n'est-il pas insuffisant pour voir le féminisme comme une expression du féminin? Il y a en effet un risque de tautologie: "les féministes sont des femmes et c'est parce qu'elles sont des femmes qu'elles sont féministes ». Sans doute faut-il examiner de plus près cette fausse évidence : être femme. Le féminisme a toujours été minoritaire parmi les femmes, et le genre n'apparaît dès lors pas comme une explication satisfaisante, ou en tout cas suffisante. Quant aux formes prises par le féminisme, elles ne relèvent à l'évidence pas d'une (éventuelle) culture féminine. Enfin, si l'on considère les positions des féministes sur les enjeux tels que la guerre et la paix, le nationalisme et l'internationalisme, la droite et la gauche, la proximité des attitudes des femmes et des hommes est frappante, la spécificité féministe apparait assez faible.

On a l'habitude de parler des féministes au féminin pluriel. Ce genre féminin, légitime compte tenu du nombre de femmes engagées dans ce combat, gomme la présence des hommes militant à leurs côtés. On ne peut que souhaiter des recherches plus approfondies sur leur histoire. I est sans doute excessif de parler d' "androgenèse " du féminisme comme le fait Philippe Régnier au sujet des saint-simoniens (il prend soin cependant d'examiner les rapports de forces : «les victoires du thème féminin sont autant de défaites des femmes dans la lutte pour le contrôle du groupe ${ }^{24}$ ). Mais on ne peut que regretter la minoration du rôle des hommes. Il n'est pas sûr qu'elle soit volontaire. Toute historiographie

23. PICQ, Françoise, Libération des femmes. Les années-mouvement, Seuil, 1993, p. 41.

24. "De l'androgenèse du féminisme : les saint-simoniens ", Cahiers du CEDREF (Études féministes de Paris VII, $\mathrm{n}^{\circ} 1,1^{\mathrm{er}}$ trimestre 1989, p. 72. 
porte la marque de son époque et le regard, même rétrospectif, porté sur les hommes féministes est sous l'influence de la radicalité féministe (surtout dans les années 1970) étroitement associée à la non-mixité. À l'époque de la première vague, qu'un homme se désigne ou soit désigné comme féministe paraissait chose normale. Vic tor Hugo, Jo hn Stuart Mill, Léon Richer, parmi bien d'autres, l'étaient ${ }^{25}$. Aujo urd'hui, la déno mination qui semble s'imposer est celle de "pro-féministe". Son usage est même rétroactif dans un des rares ouvrages historiques sur les hommes féministes: "Against the tide. Pro-feminist Men in the United States, 1776$1990 » 26$. On trouve une variante de ce scrupule dans le titre d'un article publié par l'éditeur de la revue améric aine Men and masculinities, "Qui a peur des hommes qui font du féminisme? ${ }^{27}$ : ils le font, mais ne le sont pas... Étant moi-même coupable d'avoir séparé les hommes des femmes dans le dictionnaire biographique en annexe de ma thèse distinguant d'une part les «soutiens masculins" d'autre part les «militantes féministes ", je me sens concernée par ce trouble. Jaurais dû affronter une difficulté qui n'était pas insurmontable - à savoir distinguer le soutien à la cause et le militantisme effectif - j'en ai au contraire tiré prétexte pour éviter d'intégrer les hommes dans le dictionnaire biographique des féministes. À vrai dire, cela a été inconscient, et la conscience ne m'en est venue qu'en croisant les hommes "pro-féministes » et leurs publications, quelques années plus tard. Ce qui faisait tabou, ou restait dans le domaine purement idéologique du politiquement correct hier, est en passe de devenir un débat et ne manquera pas d'attirer l'attention des historiens. Le simple choix des mots engage, suppose des choix, ou trahit des désirs inconscients. La sagesse historienne nous commande plutôt de suivre les usages de l'époque étudiée afin d'éviter tout anachronisme. En l'occurrence, le scrupule "pro-féministe", issu d'une sensibilité contemporaine (par ailleurs très discutable ${ }^{28}$ ), est déplacé pour les militants de la première vague.

Le genre est utile pour rendre compte des injustices que les féministes combattent ou la nature des oppositions qu'elles (et ils) rencontrent. Déterminisme, il est aussi un enjeu. Une manière, par exemple, de lire l'événement, à la manière de Luc Capdevila pour la masculinité au

25. Groutr, Benoîte, Le Féminisme au masculin, Denoël-Gonthier, 1977.

26. So us la direction de KIMMEL, Michael S. et MosmLLER, Thomas E. Boston, Beacon Press, 1992.

27. Dans Nouvelles approches des hommes et du masculin, sous la direction de WELZER-LANG, Daniel, Toulouse, Presses universitaires du Mirail, 2000, p. 237-254. Un o uvrage dédié "à tous les hommes, qui depuis plusieurs siècles, ont accompagné les luttes des femmes " (souligné par moi).

28. Elle indique une adhésion distanciée, justifiée par l'idée que seules les femmes parce qu'elles vivent la domination masculine peuvent être féministes. Si les bourgeois défendant la cause prolétaire avaient eu les mêmes scrupules, le prosocialisme aurait été un mouvement de masse ! Plus sérieusement, on est confronté aussi à un problème étymologique : féminisme est construit à partir du mot femme, ce qui n'aide pas les hommes à se sentir eux aussi concernés. On voit aujourd'hui combien l'antisexisme facilite l'adhésion masculine. 
moment de la Libération ${ }^{29}$. Ou une manière de lire l'histo ire du féminisme dont la raison d'être, l'égalité, interroge, dérange, déstabilise les identités de genre. L'indifférenciation plane comme une menace pour tous ses détracteurs ${ }^{30}$, mais gêne et piège la plupart des féministes qui répondent "féministes oui, mais féminines ". Pourquoi, alors que Virginia Wo olf écrivait Orlando et que Claude Cahun se photographiait le crâne rasé, Violette Morriss faisait réduire sa poitrine pour mieux conduire son bolide, les féministes étaient-elles si timorées? La force subversive du pouvoir culturel semble plus agissante que celle du pouvoir politique, lorsque les codes de genre ou les normes sexuelles sont en jeu ${ }^{31}$.

\section{La hantise de la masculinisation des femmes}

La crainte de la masculinisation des femmes n'est pas une anecdote dans l'histoire du féminisme et de l'antiféminisme. On peut penser, si l'on s'inscrit dans la tradition d'une analyse matérialiste des rapports de sexe, que la polémique sur les identités de genre a une fonction de brouillage des enjeux réels, concrets. Beaucoup de féministes ont raiso nné et raisonnent to ujours ainsi : l'identité relèverait de préoccupations superficielles, voire oiseuses. Ainsi, la plupart des féministes de la première vague ont soin de placer l'égalité des sexes sur le terrain du Droit, lequel doit ignorer le sexe (sauf pour ce qui concerne la maternité). Les féministes de la seconde vague héritières de Simone de Beauvoir évacuent aussi l'identité, dans le sens où elles en font le reliquat pénible de la différenciation sociale des sexes. Elles se concentrent au contraire sur les aspects matériels de l'oppression (voir, par exemple, l'analyse de Christine Delphy sur l'exploitation domestique ${ }^{32}$ ). Mais lorsque les féministes "de l'égalité » délaissent la réflexion sur l'identité, le terrain est occupé par des féministes qui ne partagent pas leur crainte de l'essentialisme et/ou du naturalisme. Ces dernières, depuis fort longtemps, proposent des «féminitudes " qui dessinent un "destin » féminin lié au corps et à la maternité.

L'épisode de la garçonne, mode marquante des années 1920, est très révélateur des craintes des féministes. Comme l'indique le mot "garçonne », popularisé par un homme, Victor Margueritte, le référent de cette nouvelle "identité féminine " est masculin. La jeune fille "moderne " agit «comme un homme». Elle profite de la même liberté de mœurs et chérit son auto nomie individuelle. Elle porte des tailleurs qui effacent ses

29. CAPDEVILA, Luc, «Le mythe du guerrier et la construction sociale d'un "éternel masculin " après la guerre ", Revue française de psychanalyse, $\mathrm{n}^{\circ}$ 2, 1998, p. 607-623.

30. C'est un lancinant refrain dans les études rassemblées dans Un siècle d'antiféminisme, Fayard, 1999.

31. Marie-Jo Bonnet formule ce constat en examinant l'histoire des lesbiennes qui s'expriment (jusqu'aux années 1970) dans la sphère culturelle mais pas dans la sphère politique (BONNET, Marie-Jo, Les Relations amoureuses entre les femmes du XVI $t^{e}$ au XX siècle, Odile Jac ob, 1995).

32. DELPHY, Christine, L'Ennemi principal, Syllepse, 1998. 
courbes; ses cheveux sont courts; elle conduit des bolides et fume en public. Cette image de mode largement exploitée par les romanciers reflète pour une part des évolutions réelles. Il est cependant excessif d'y voir un triomphe de la virilisation des femmes. C'est pourtant bien ce que beaucoup d'auteurs antiféministes ont voulu faire en condamnant la garçonne, femme virilisée et contre-nature, victime de l'émancipation des femmes. Rien de bien surprenant. En revanche, que les féministes - à de très rares exceptions près - aient condamné, elles aussi, la garçonne ne peut que nous intriguer. Les cris d'horreur provoqués par les atteintes à la «féminité " méritent que l'on explore des raisons plus profondes encore. Elles traduisent sans doute la crainte d'une déstabilisation de l'ordre hétérosexuel. Les codes de la féminité se confondent avec les codes de la séduction hétéro sexuelle. La remise en question de ces codes renvoie donc presque auto matiquement à ce que l'on a longtemps appelé l'inversion sexuelle. Or cette transgression est spontanément perçue comme "féministe". La lesbophobie, source d'inspiration pour les antiféministes, est une source de gêne pour les féministes. Il faudrait sans doute réfléchir davantage au rapport des femmes - et des féministes - à la masculinité. Le sujet est comme tabou, et encore nimbé de mystère. Interdit secrètement convoité (porter le pantalon), fascination indicible (surto ut quand on est féministe!), sentiment de trahison? Et si les emprunts au vestiaire masculin (phénomène majeur $a u x^{\mathrm{e}}$ siècle), très visibles, cachaient mille et une appropriations du masculin?

Aujourd'hui, nous sommes accoutumés à l'image de femmes militaires, nous rions avec Josiane Balasko (et non de) en regardant Gazon Maudit. Je cite à dessein les deux images les plus fortes de la «masculinité féminine ${ }^{33}$ : la femme en uniforme et en armes, et la lesbienne virile, plus couramment appelée "camionneuse ". Dans un récent (2000) "Arrêt sur images ", où Roselyne Bachelot et Clémentine Autain (co-présidente de Mix'Cité) étaient invitées, après des commentaires pertinents sur l'image obligée d'hyperféminité que doivent donner à la télévision les présentatrices et les femmes journalistes, un extrait de bulletin d'information est montré, correspondant bien à la tendance paritariste actuelle: une femme et un homme présentent ensemble le journal. Mais la première porte, comme son collègue, des cheveux courts, une chemise et une large cravate. Question du journaliste : Alors, c'est ça que vous voulez?" Réponse des invitées : "Non, là c'est ridicule».

Ce commentaire lapidaire montre la force incroyable de cette crainte de la virilisation des femmes, même, nous l'avons souligné chez les féministes. Le look étant chose sérieuse à la télévision mais aussi en politique,

33. Je reprends ici l'expression de Judith HALBERSTAM, Fe male Masculinity, Durham and London, Duke University Press, 1998 qui étudie les diverses formes de masculinité déclinées par des femmes depuis deux cents ans, en se centrant plus particulièrement sur les lesbiennes «viriles", «travesties » ou «transgenres ». 
en particulier pour les femmes comme l'a bien montré Jane Freedman ${ }^{34}$, il faut sans doute chercher ailleurs les explications à cette remarque qui pouvait être perçue comme désobligeante à l'égard de cette journaliste. Ro selyne Bachelot est à cet égard intéressante car elle ne fait pas mystère de sa stratégie vestimentaire. Si elle porte des tailleurs aux "couleurs pétantes ", c'est pour signaler qu'elle est une femme (dans un monde d'hommes). Elle a d'ailleurs après les dernières élections législatives critiqué les femmes de gauche, arrivées à l'assemblée parées de toutes les couleurs des "oiseaux du paradis" (pour reprendre son expression) et vite passées à l'uniforme grisâtre (et masculin) de règle dans l'hémicycle. Malgré ces signes visibles de "féminité ", Roselyne Bachelot a une image publique ambivalente: sa forte voix très assurée et son franc-parler incluant l'usage de mots que certains trouvent grossiers peuvent être vus comme masculins. Une des clés de son succès réside peut-être dans cet art de mixer les genres : cette fameuse androgynie qui ne manque pas de fasciner jusqu'aux esprits les plus conformistes. La politique, le pouvoir, le féminisme ne sont pas seulement des discours, mais des images et des projections de notre imaginaire, et leur dimension érotique gagnerait à être mieux explorée.

\section{RÉSUMÉ}

La notion de genre est ici examinée à travers quelques unes de ses applications possibles en histoire du féminisme. Produite par les féministes, elle permet de dévoiler la répartition inégale du pouvoir entre les sexes. Elle peut aussi éclairer certains aspects de l'histoire interne du féminisme. Elle est surtout cruciale dès lors que l'on s'intéresse à la conscience des sujets. Enfin, on verra comment les normes de genre affectent les transformations identitaires à travers la phobie persistante de la «virilisation des femmes».

\section{ABSTRACT}

The notion of gender is studied here through several possible applications to the history of feminism. A feminist production, this notion reveals the uneven distribution of power between the sexes, and can also shed light on certain aspects of the internal history of feminism. It is especially crucial for the study of subjects' awareness. Finally, the effect of gender norms on identity-based transformations, through a persistent fear of the masculinisation of women, will be examined.

34. FREEDMAN, Jane, Femmes politique s: mythe s et symboles, L'Harmattan, 1997. 